\title{
Telemedicine in the COVID-19 era: the new normal
}

\author{
Praveen Kumar · Farhanul Huda · Somprakas Basu
}

Received: 15 August 2020 / Accepted: 1 September 2020 / Published online: 8 October 2020

(C) Springer-Verlag GmbH Austria, part of Springer Nature 2020

\section{Dear Editor,}

It is now known that COVID-19 is spreading through aerosolized droplets. In order to avoid face-to-face contact without hampering healthcare provision, telemedicine (TM), which is now a part of the digital health systems of various countries, needs to be applied more effectively. Except for acute cases, chronic conditions and follow-up visits can be managed using $\mathrm{TM}$, and thus unnecessary hospital visits are reduced while ensuring triage and treatment of the seriously ill. The World Health Organization (WHO) considers TM as a mode of healthcare delivery when the distance is a critical factor [1]. It is, therefore, essential to learning information and communication technologies for the exchange of valid information. In this pandemic, many boundaries have been pushed back and opportunities are constantly being explored. The application of TM embarks in areas that were once considered potentially unsafe for its use in healthcare; however, the lack of uniform legislation to integrate TM into healthcare is a significant challenge in its extended use in the current pandemic [2].

In order to be effective, health providers should have the necessary education, licensure, and professional capacity to deliver healthcare services through TM. In order to expand its use, strict regulations on the requirements for the establishment of the patient-provider relationship and credentialing have been waived on many occasions. Direct access testing laws have allowed laboratories to do diagnostic tests following teleconsultation with the physician [3]. The United States (US) Drug Enforcement Administration allowed prescribing controlled substances with tele-

P. Kumar · F. Huda · S. Basu ( $ه)$

Department of Surgery, All India Institute of Medical

Sciences, Rishikesh, India

sombasu@hotmail.com consultation, in an audio-visual, real-time two-way communication mode [4]. Even informed consent has been taken through TM [5]. In these circumstances, it is challenging to maintain records, privacy, and confidentiality. Platforms such as Messenger, Video Chat, Google Video, and Skype have been used according to new regulatory flexibilities in TM [6].

TM can be used to forward triage before a patient reaches the hospital [7]. Triage is done in two phases, firstly a telephone triage for potential COVID-19 cases or contacts followed by an in-office triage that identifies cases which were in the incubation period at the time of telephone triage and developed symptoms before the in-office visit. Chronic stable diseases such as diabetes, COPD, hypertension, and immunodeficiency diseases can be managed by TM without increasing the risk of complications or exposure. It decreases the cost of follow-up visits, and no difference in the treatment of chronic conditions by TM over inperson visits has been observed.

Medical teaching has mainly been shifted to online formats through interactive seminars, group discussions, and clinical skill sessions [8]. Students can perform clinical tasks with the help of trained health aides, thereby slowly replacing a teacher's role as a mentor in the virtual environment. The online surgical course can be conducted where students can be tele-mentored. Although surgeries can be demonstrated live and discussion among participants at different places is possible, direct patient interaction, clinical examination, and elicitation of physical signs are not possible in a virtual platform. Also, the conduction of online practical examination lacks a full evaluation of competencies. The traditional surgical training where residents learn by performing under supervision remains wanting.

Empathy in the provider-patient relationship, which increases patient compliance and faith, is difficult to 
demonstrate through TM. The principal reason is the online disinhibition effect [9]. It is a state where a person psychologically disconnects from their actual being when they are not facing one another. There are three possible ways to enhance empathy during teleconsultations. First is active listening and to let the patient guide the conversation. The second is to follow up after the appointment through text messages and emails so that the patient does not feel forgotten. The third is to personalize communication through psychographic segmentation. Psychologically speaking, patients can be self-achievers and wilful endurers. Self-achievers are health-conscious, whereas wilful endurers tend to neglect their health. A TM consultant should identify this psychological capacity and act accordingly. The TM consultants should be trained to improve communication skills to make a useful TM encounter.

However, TM in healthcare is not without limitations. Imposters can represent real patients. In video consultations, there is a possibility of misuse of privacy of patients. In asynchronous communication such as emails, there will be a delay in response. Prescribing medications on telecommunication may invite errors. One health survey stated that reimbursement and licensing requirements were significant challenges [10]. Moreover, any breach in technology can lead to loss of patient's privacy and confidentiality.

When used strategically, TM can be a powerful tool for education and healthcare delivery in the current pandemic. In order to utilize it to its fullest potential, legal issues need to be addressed to meet the current need. Further training of healthcare workers is required for a better outcome.

Conflict of interest S. Basu declares that there are no competing interests.

\section{References}

1. WHO Global Observatory for eHealth. TM: opportunities and developments in Member States: report on the sec- ond global survey on eHealth[Internet]. 2010. Available from: https://apps.who.int/iris/handle/10665/44497. Last access: 2020 Aug 02.

2. Smith AC, Thomas E, Snoswell CL, et al. Telehealth for global emergencies: implications for coronavirus disease 2019 (COVID-19). J Telemed Telecare. 2020;26(5):309-13. https://doi.org/10.1177/1357633X20916567.

3. Arnold, Porter. Telehealth during COVID 19: new rules and considerations [Internet]. 2020. https://www.mondaq. com/unitedstates/healthcare/922532/telehealth-duringcovid-19-new-rules-and-considerations. Last access: 2020Aug02

4. Kannarkat JT, Smith NN, McLeod-Bryant SA. Mobilization of telepsychiatry in response to COVID-19-moving toward 21st century access to care. Adm Policy Ment Health. 2020;47(4):489-91. https://doi.org/10.1007/s10488-02001044-z.

5. American Society of Plastic Surgeons. Informed consent:telemedicine[Internet]. 2020. https://www. plasticsurgery.org/documents/medical-professionals/

Telemedicine-Informed-Consent.pdf. Last access: 2020 Sep 23.

6. Pool MM, Saul HC. HIPAA waivers and compliance in COVID-19 pandemic [Internet]. 2020 March 17. Available from: https://www.agg.com/news-insights/publications/ hipaa-waivers-and-compliance-in-covid-19-pandemic/. Lastaccess: 2020 Aug 04.

7. Cervino G, Oteri G. COVID-19 pandemic and telephone triage before attending medical office: problem or opportunity? Medicina (Kaunas). 2020;56(5):250. https://doi.org/ $10.3390 /$ medicina56050250.

8. Mukundan S Jr, Vydareny K, Vassallo DJ, Irving S, Ogaoga D. TrialTM system for supporting medical students on elective in the developing world. Acad Radiol. 2003;10(7):794-7. https://doi.org/10.1016/s1076-6332(03)80125-3.

9. Terry C, Cain J. The Emerging Issue of Digital Empathy. Am J Pharm Educ. 2016;80(4):58. https://doi.org/10.5688/ ajpe80458.

10. Lacktman NM, Rosen DL, Chmielewski MR, Beaver NA. 2017 Telemedicine \& Digital Health Survey [Internet]. 2017 Nov 15. Available from: https://www.foley.com/ en/insights/publications/2017/11/2017-telemedicine-digital-health-survey. Last access: 2020 Aug 6.

Publisher's Note Springer Nature remains neutral with regard to jurisdictional claims in published maps and institutional affiliations. 